\title{
Is Body Mass Index for-age Related with Dental Caries in Children?
}

\author{
(1) Esra Kızılcı1, (1) Cansu Demiroğlu1', (1) Burcu Duman2, (1) Büşra Ayhan3 \\ ${ }^{1}$ Erciyes University Faculty of Dentistry, Department of Pediatric Dentistry, Kayseri, Turkey \\ 2Private Çorum Denta Gross Oral and Dental Health Center, Çorum, Turkey \\ ${ }^{3}$ Gazi University Faculty of Health Sciences, Department of Nutrition and Dietetic, Ankara, Turkey
}

\begin{abstract}
Aim: Nutrition has a significant impact on dental health. The aim of this study was to investigate the relationship between dental caries and body mass index (BMI)-for-age.

Materials and Methods: Four hundred and fifty-one children who were 5-14 years were included in this study. The children were examined according to dmft/DMFT indexes with mirror - sound and panoramic radiography. After taking weight and height measurements, the BMIfor-age calculation, age, weight and height status were entered into the World Health Organization AnthroPlus Software program in order to obtain a z-score for each child. The children were grouped according to their z-scores as Group 1: normal; Group 2: overweight; Group 3: fat/obese; Group 4: underweight; and Group 5: severely underweight. Data were analyzed using SPSS software 22, correlation, frequency and independent sample t-tests.
\end{abstract}

Results: The mean age of the children was $9.1 \pm 2.5$ years. The mean DMFT/dmft values were $6.97 \pm 3.79 .55 \%$ of the children were of normal weight, $16.2 \%$ were overweight, $11.8 \%$ were fat/obese, $12.2 \%$ were underweight and $4.8 \%$ were severely underweight. In terms of DMFT/dmft, when the groups were compared, a statistically significant difference was found between Group 3 and Groups 1, 4 and 5 ( $p<0.05$ ).

Conclusion: The risk of caries increased as the weight increased in the children participating in this study. Obesity can be considered as an important risk factor for dental health. Informing parents about children's poor eating habits can have an impact on dental health.

Keywords: Dental caries, body mass index by age, obesity

\section{Introduction}

Proper nutrition has a significant impact on oral and dental health as well as on physical and cognitive health $(1,2)$. Dental caries are a multifactorial disease that is caused by cariogenic bacteria, a carbohydrate-rich diet and high host susceptibility (3-5).

Obesity and tooth decay have common and variable features such as diet and lifestyle (1). Being overweight and obesity are among the health problems that are increasing in our country as well as worldwide (6). Today, obesity, which is increasing rapidly in adults and adolescents, is called "obeziteus" in Latin and means "due to eating" (7). It is a multifactorial disease characterized by stored fat as a result of an increase in the amount of fatty and carbohydrate foods consumed resulting from having more calories entering the body than the number of calories expended (8). It is also known as an energy metabolism disorder that can cause physical and psychological problems $(6,9)$. The main causes of obesity, defined by the World Health 
Organization (WHO) as a global epidemic, are considered to be environmental factors such as practical living conditions, immobility, eating habits, in addition to genetic and physical factors (7).

The nutritional habits of children are among the most important causes of both childhood obesity and dental caries. Some children who experience early tooth loss may face problems such as weight gain due to incomplete digestion resulting from a decrease in chewing function (1). Tooth loss and caries may also be associated with both obesity and weakness. Weight loss can be seen in children who have difficulty feeding due to early tooth loss and painful teeth. Severe dental caries can reduce the action of eating and thus cause weight loss (10).

Among the obesity criteria of the $\mathrm{WHO}$ is the body mass index (BMI), also called the Quetelet index. BMI is calculated by dividing an individual's body weight by the square of his/ her height $\left(\mathrm{BMI}=\mathrm{kg} / \mathrm{m}^{2}\right)$. The classification of children and adolescents is different from adults and "z-score" values are used (4). As growth and development continues in children, body fat changes; therefore, the assessment of $\mathrm{BMI}$ in children and adolescents is made by BMI-for-age; those who are in the $5^{\text {th }}$ percentile and below are considered as "underweight", those who are in the $85^{\text {th }}$ percentile and above are at risk of being "overweight" and those who are in the $95^{\text {th }}$ percentile and above are considered as "overweight" (11).

In many studies relating to obesity and dental caries, the relationship between $\mathrm{BMI}$ and caries in the child age group has been evaluated $(2,10,12-14)$. García Pérez et al. (15) used $\mathrm{BMI}$-for-age in their study and examined the relationship between the caries experience and obesity in Mexican 8-12 year-old school children. The aim of the current study was to investigate the relationship between dental caries and BMI-for-age (normal, overweight, obese, underweight and severely underweight) in a group of 5-14 year-old Turkish children. Secondly, it was hypothesized that the results obtained from evaluating caries with BMI-for-age might be different from results obtained using BMI.

\section{Materials and Methods}

According to the power analysis, the $\mathrm{n}$ value was calculated to be 451 with a confidence interval of $\alpha=0.05$ and $\beta=0.95$. Those children between the ages of $5-14$ who applied to the Department of Pedodontics in Erciyes University were included in this study. Ethics committee approval was received for this study from Erciyes University Faculty of Medicine (approval no: 2020/60, date: 29.01.2020).
Those patients with systemic diseases which may affect growth and development were excluded from this study. After obtaining consent forms from the patients and their parents stating that they want to participate in the study, the demographic information (age, gender, etc.) of the patients was recorded.

\section{Study Design}

The participants were asked to remove their shoes in order to record their body weight and height measurements; a Tanita ${ }^{\circledR}$ scale (Bc601 model) with integrated stadiometer was used to perform both measurements. The BMI-for-age calculation was made by entering the age, weight and height measurements into the WHO AnthroPlus Software program and the $z$-score was obtained. The children were grouped according to their z-scores as Group 1: normal, Group 2: overweight, Group 3: fat/obese, Group 4: underweight, and Group 5: severely underweight. The calculations of anthropometric evaluations were made by a nutritionist and dietetics specialist.

Intraoral examination was made after the teeth were air-dried by a research assistant dentist with the help of a mouth mirror and dental probe. The measurements were recorded by another dentist. According to the criteria determined by the WHO, the DMFT (decay, missing, and filled teeth) index was used to determine the number of caries in permanent teeth and the $\mathrm{dmft}$ index was used in primary teeth. The existing periapical and panoramic radiographs of the patients were used in the diagnosis of interface caries.

The data were analyzed by correlation, frequency and independent sample t-tests using the IBM SPSS Statistic (v.22) software.

\section{Results}

The average age of the children who participated in this study was $9.1 \pm 2.5$ years. Of these, $52 \%(n=234)$ were female and $48 \%(n=217)$ were male. Average DMFT/dmft values were found to be $6.97 \pm 3.79$. It was found that 425 of the children in the study group had at least 1 caries. The caries percentages of the females and males were $91 \%$ and $97 \%$, respectively. There was no significant difference in the number of caries between the two groups ( $p>0.05)$. When the children were grouped according to their BMI-for-age values, $55 \%$ of them were found to be normal $(n=248)$, $16.2 \%$ were overweight $(n=73), 11.8 \%$ were obese $(n=53)$, $12.2 \%$ were underweight, $(n=55)$ and $4.8 \%$ were severely underweight $(n=22)$. Overweight and obese participants constituted $24 \%$ of the group. $45 \%(n=24)$ of Group 3 
were girls and $54 \%(n=29)$ were boys (Table I). There was no significant relationship between gender and obesity ( $p>0.05)$. In terms of the DMFT/dmft index, when groups were compared according to their BMI-for-age (Graph 1), no difference was observed between obese participants and overweight participants ( $p>0.05)$. A statistically significant difference was found between Group 3 and the other 3 groups (Group 1, Group 4 and Group 5) $(p<0.05)$. There was no significant difference observed when the other groups were compared with each other $(p>0.05)$. No relation was

Table I. The frequency and percentage distribution of children based on BMI-for-age

\begin{tabular}{|c|c|c|c|}
\hline \multicolumn{2}{|c|}{ BMI-for-age } & \multirow{2}{*}{$\begin{array}{l}\mathbf{n} \\
131\end{array}$} & \multirow{2}{*}{$\begin{array}{l}\% \\
52.8\end{array}$} \\
\hline \multirow{3}{*}{ Group 1} & Female & & \\
\hline & Male & 117 & 47.2 \\
\hline & Total & 248 & 100 \\
\hline \multirow{3}{*}{ Group 2} & Female & 35 & 47.9 \\
\hline & Male & 38 & 52.1 \\
\hline & Total & 73 & 100 \\
\hline \multirow{3}{*}{ Group 3} & Female & 24 & 45.3 \\
\hline & Male & 29 & 54.7 \\
\hline & Total & 53 & 100 \\
\hline \multirow{3}{*}{ Group 4} & Female & 33 & 60 \\
\hline & Male & 22 & 40 \\
\hline & Total & 55 & 100 \\
\hline \multirow{3}{*}{ Group 5} & Female & 11 & 50 \\
\hline & Male & 11 & 50 \\
\hline & Total & 22 & 100 \\
\hline
\end{tabular}

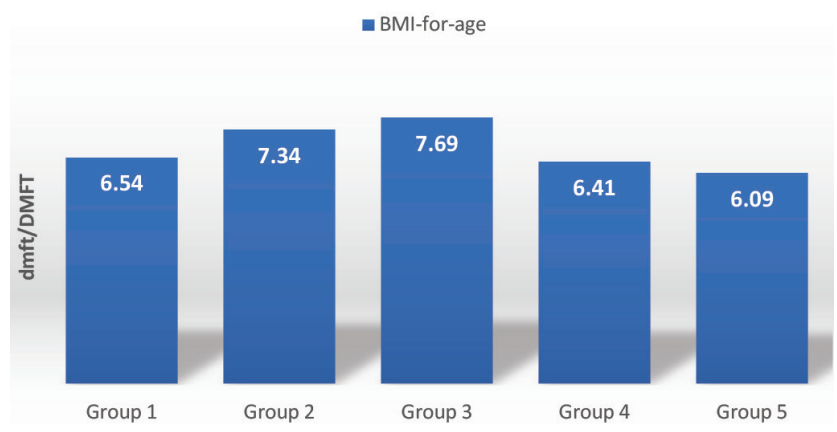

Graph 1. Diagram showing mean DMFT/dmft among children with different body weights

Group 1: Normal, Group 2: Overweight, Group 3: Fat/obese, Group 4: Underweight, Group 5: Severely underweight ${ }^{a-b}$ Different letters denote the statistical differences $(p<0.05)$ observed between the risk of caries and being underweight $(p>0.05)$. The risk of caries increased as weight increased in those children who participated in this study.

\section{Discussion}

In recent years, there has been an increase in overweight and obesity rates due to an increase in over-nutrition habits of children. It has also started to come into prominence in studies investigating the relationship between tooth decay and weight, which are both closely related to dietary habits $(14,16,17)$.

Obesity and overweight status can be measured using the BMI. However, BMI is calculated by dividing body weight $(\mathrm{kg})$ by the square of the height $(\mathrm{m})$, and it does not take into account variable values such as age, gender, and body fat percentage (18). Since growth is on-going in children and adolescents, body fat is in the process of change; for this reason, the evaluation of $\mathrm{BMI}$ in children and adolescents according to age will give more accurate results. Therefore, in our study, the BMI-for-age calculation was made by obtaining z-scores by entering age, weight and height measurements into the AnthroPlus Software, as recommended by the WHO for children under the age of 18 years. In many studies, only obesity or severely underweight conditions were evaluated $(10,15,19,20)$. In our study, not only obese and overweight children, but also underweight, severely underweight and normal children were included in the evaluation.

A study in Mexico found that approximately one-fifth of students were obese, and the proportion of overweight and obese children constituted $37.7 \%$. of the whole group (15). In our study, $11.8 \%$ of the children who participated were found to be obese, and the overweight and obese groups together constituted $24 \%$. Comparing these two studies, the lower values obtained in our study may stem from different eating habits within our country. We believe that it is possible to provide data to explain any differences by an evaluation of the nutritional habits of children by dietetic and nutritionist specialists.

Bafti et al. (8) found that tooth decay in the primary teeth of children was inversely proportional to an increase in their body weight. In another study, Benzian et al. (10) investigated the relationship between $\mathrm{BMI}$ and dental caries in underweight children and found a significant correlation between $\mathrm{BMI}$ and dental caries in low-weight children. On the other hand, Mitrakul et al. (19) and Dikshit et al. (14) argued that there was no significant relationship between dental caries and BMI. 
In many studies, the risk of dental caries was found to be higher in overweight and obese children (21-23). Cheng et al. (24) showed that the frequency of dental caries among primary and secondary school children is related to gender, school type, region and BMI. They claimed that extensive studies are needed to confirm the findings of their study and to address existing oral health problems (24). The study conducted by Barrington et al. (25) focused on the incidence of the relationship between obesity and caries. However, they emphasized that sugar and carbohydrate consumption status are factors that should be evaluated primarily for both (25).

Karki et al. (16) declared that children with low or high $\mathrm{BMI}$, regardless of their age, are at risk of developing caries. They emphasized that both underweight and overweight or obese children have common risk factors relating to their diet (16).

Another related study conducted by Willerhausen et al. (2) also found a significant relationship between being overweight and caries. According to the results of our study, a positive relationship was found between obesity and caries and these results support the findings of the study conducted by Willerhausen et al. (2).

In addition, in the study conducted by García Pérez et al. (15), in which BMI-for-age evaluations were used as in our study, it was reported that there was an inversely proportional relationship between obesity and caries as was also seen in other studies using $\mathrm{BMI}(8,10)$. However, it was emphasized that caries is a complex process including a contribution of various factors such as fluoride consumption, sugar intake, and brushing teeth, none of which should be ignored (18). In our results, there was no significant relationship between underweight children and the incidence of caries, which is in contrast to the results given by García Pérez et al. (15). We presume that this situation may be due to the low number of underweight children in our study group or to different eating habits among countries.

However, as mentioned in the results of some studies, explaining the relationship between caries and BMI by various factors such as poor eating habits, frequency and times of sugar consumption, malnutrition, frequency of brushing teeth, fluoride intake or a sedentary lifestyle is a complex process $(26,27)$.

\section{Study Limitations}

One of the limitations of this study was that the sugar and carbohydrate consumption of children was not evaluated together with BMls-for-age. The small sample size for severely underweight children was another limitation for our study.

Nkambule et al. (17) stated that the incidence of caries is associated with an availability of access to health services rather than to other factors. In our study, we tried to eliminate this parameter by means of a working group who could access health services in our clinic. However, it should not be forgotten that field studies may reveal different results.

\section{Conclusion}

Why this paper is important to pediatric dentists/ pediatricians:

- Within the limitations of this study, no relationship between the risk of caries and being underweight was found.

- Obesity was found to be an important risk factor for oral and dental health.

- Parents should be informed that their child's bodymass-index for age has an impact on oral and dental health.

\section{Ethics}

Ethics Committee Approval: This study was approved by the ethics committee of Erciyes University, Faculty of Medicine Clinical Research Ethics Committee (number: 2020/60, date: 29.01.2020).

Informed Consent: Informed consent was obtained from all of the parents included in the present study.

Peer-review: Externally peer-reviewed.

\section{Authorship Contributions}

Concept and Design: E.K., C.D., B.D., B.A., Data Collection or Processing: C.D., B.D., Analysis Interpretation: E.K., B.A., Drafting of Manuscript: E.K., C.D., B.D., B.A., Critical Revision: E.K.

Conflict of Interest: The authors declare that they have no conflict of interest.

Financial Disclosure: The authors declared that this study received no financial support.

\section{References}

1. Hooley M, Skouteris H, Boganin C, Satur J, Kilpatrick N. Body mass index and dental caries in children and adolescents: a systematic review of literature published 2004 to 2011. Syst Rev 2012; 1:57.

2. Willerhausen B, Blettner M, Kasaj A, Hohenfellner K. Association between body mass index and dental health in 1,290 children of elementary schools in a German city. Clin Oral Investig 2007; 11:195-200. 
3. Sheiham A, James WP. Diet and Dental Caries: The Pivotal Role of Free Sugars Reemphasized. I Dent Res 2015; 94:1341-7.

4. Pitts NB, Zero DT, Marsh PD, et al. Dental caries. Nat Rev Dis Primers 2017; 3:17030.

5. Hilgers KK, Kinane DE, Scheetz JP. Association between childhood obesity and smooth-surface caries in posterior teeth: a preliminary study. Pediatr Dent 2006; 28:23-8.

6. Lee EY, Yoon $\mathrm{KH}$. Epidemic obesity in children and adolescents: risk factors and prevention. Front Med 2018; 12:658-66.

7. No authors listed. Obesity: preventing and managing the global epidemic. Report of a WHO consultation. World Health Organ Tech Rep Ser 2000; 894:1-253.

8. Bafti LS, Hashemipour MA, Poureslami H, Hoseinian Z. Relationship between Body Mass Index and Tooth Decay in a Population of 3-6-Year-Old Children in Iran. Int I Dent 2015; 2015:126530.

9. Samdal GB, Eide GE, Barth T, Williams G, Meland E. Effective behaviour change techniques for physical activity and healthy eating in overweight and obese adults; systematic review and meta-regression analyses. Int / Behav Nutr Phys Act 2017; 14:42.

10. Benzian H, Monse B, Heinrich-Weltzien R, Hobdell M, Mulder I, van Palenstein Helderman W. Untreated severe dental decay: a neglected determinant of low Body Mass Index in 12-year-old Filipino children. BMC Public Health 2011; 11:558.

11. WHO Multicentre Growth Reference Study Group. WHO Child Growth Standards based on length/height, weight and age. Acta Paediatr Suppl 2006; 450:76-85.

12. Basha S, Mohamed RN, Swamy HS, Ramamurthy PH, Sexena V. Caries Incidence Among Obese Adolescents: A 3-year Prospective Study. Oral Health Prev Dent 2017; 15:65-71.

13. Cinar AB, Christensen LB, Hede B. Clustering of obesity and dental caries with lifestyle factors among Danish adolescents. Oral Health Prev Dent 2011; 9:123-30.

14. Dikshit P, Limbu S, Bhattarai R. Relationship of Body Mass Index with Dental Caries among Children attending Pediatric Dental Department in an Institute. INMA / Nepal Med Assoc 2018; 56:582-6.

15. García Pérez A, Barrera Ortega CC, González-Aragón Pineda ÁE, Villanueva Gutiérrez T, Pérez Pérez NG, Calderon Uriostegui D. An inverse relationship between obesity and dental caries in Mexican schoolchildren: a cross-sectional study. Public Health 2020; 80:163-7.
16. Karki S, Päkkilä J, Ryhänen T, et al. Body mass index and dental caries experience in Nepalese schoolchildren. Community Dent Oral Epidemiol 2019; 47:346-57.

17. Nkambule NR, Madiba TK, Bhayat A. Dental Caries, Body Mass Index, and Diet among Learners at Selected Primary Schools in Pretoria, Gauteng Province, South Africa. I Contemp Dent Pract $2019 ; 20: 1241-8$

18. Cantekin K, Gurbuz T, Demirbuga S, Demirci T, Duruk G. Dental caries and body mass index in a sample of 12-year-old eastern Turkish children. J Dent Sci 2012; 7:77-80.

19. Mitrakul K, Arunakul M, Asvanund $Y$, Laisirireoungrai $T$, Praneechotiros T, Tevavichulada P. Diet, Body Mass Index And Dental Caries Among Thai Children Aged 3 To 5 Years. Southeast Asian J Trop Med Public Health 2017; 48:466-72.

20. Serrano-Piña R, Aguilar-Ayala FJ, Scougall-Vilchis RJ, TrujilloGüiza ML, Mendieta-Zerón H. Prevalence of Obesity in Elementary School Children and its Association with Dental Caries. Oral Health Prev Dent 2020; 18:35-42.

21. Costacurta M, Di Renzo L, Bianchi A, Fabiocchi F, De Lorenzo A, Docimo R. Obesity and dental caries in paediatric patients. A cross-sectional study. Eur J Paediatr Dent 2011; 12:112-6.

22. Gerdin EW, Angbratt M, Aronsson K, Eriksson E, Johansson I. Dental caries and body mass index by socio-economic status in Swedish children. Community Dent Oral Epidemiol 2008; 36:459-65.

23. Sharma A, Hegde AM. Relationship between body mass index, caries experience and dietary preferences in children. I Clin Pediatr Dent 2009; 34:49-52.

24. Cheng YH, Liao Y, Chen DY, Wang Y, Wu Y. Prevalence of dental caries and its association with body mass index among schoolage children in Shenzhen, China. BMC Oral Health 2019; 19:270.

25. Barrington G, Khan S, Kent K, Brennan DS, Crocombe LA, Bettiol S. Obesity, dietary sugar and dental caries in Australian adults. Int Dent ) 2019; 69:383-91.

26. Palacios C, Rivas-Tumanyan S, Morou-Bermúdez E, Colon AM, Torres RY, Elías-Boneta AR. Association between Type, Amount, and Pattern of Carbohydrate Consumption with Dental Caries in 12-Year-Olds in Puerto Rico. Caries Res 2016;50:560-70.

27. Trumbo PR, Rivers CR. Systematic review of the evidence for an association between sugar-sweetened beverage consumption and risk of obesity. Nutr Rev 2014; 72:566-74. 